\title{
水在套管内的湍流换热
}

\section{颜迪民许国华 董振武胡克杰}

目前关于套管内流体的湍流换热还存在 着很大的分歧. 有的认为双面加热与单面加 热时的放热系数一样, 有的认为放热系数与 内、外管的热负荷比值有关. Jordan 还提出 了热当量直径方法, 用来考虑加热周边的比 例．在参考资料 [1]中,我们曾对平行板及同 心套管内流体放热规律作了理论研究. 计算 结果表明, 两壁热负荷比值对传热有明显的 影响, 但随着 $P_{r}$ 数的增加而减小. 本文将对 套管内水的放热进行试验研究, 重点是两壁 热负荷比值的影响。

\section{一、试 验方法}

试验在三个试验本体上进行, 其主要几 何尺寸及工作参数列于表 1. 试验本体由试 验元件(包括内、外套管)、试验本体外壳、定 位环及绝缘密封装置组成. 图 1 表示了 1 \# 本体的结构图, $2^{\#} 、 3^{\#}$ 本体结构较简单, 图 上没表示. 1 \#本体采用薄壁管的目的是减小 壁温降, 以提高实验精确度. 但薄壁不能承 受外压, 为此设置了压力平衡系统. 元件加 热电流由两台 200 既直流发电机供给, 每台 最大电流为 10,000 安. 试验在中温中压水 迴路上进行.

试验时测量下列各参数: 试验本体进出 口水温, 经过试验本体的流量, 加热元件上的 电流、电压, 迴路压力, 内外套管的壁面温度 等.

内套管沿轴向安装了十二副镍铬-锦铝 热电偶, 其中有六副在计算截面上, 以测定其 周向温度. 为此专门设计了一套测温装置, 用直径 0.2 毫米的镍铬一镍铝热电偶作温度测

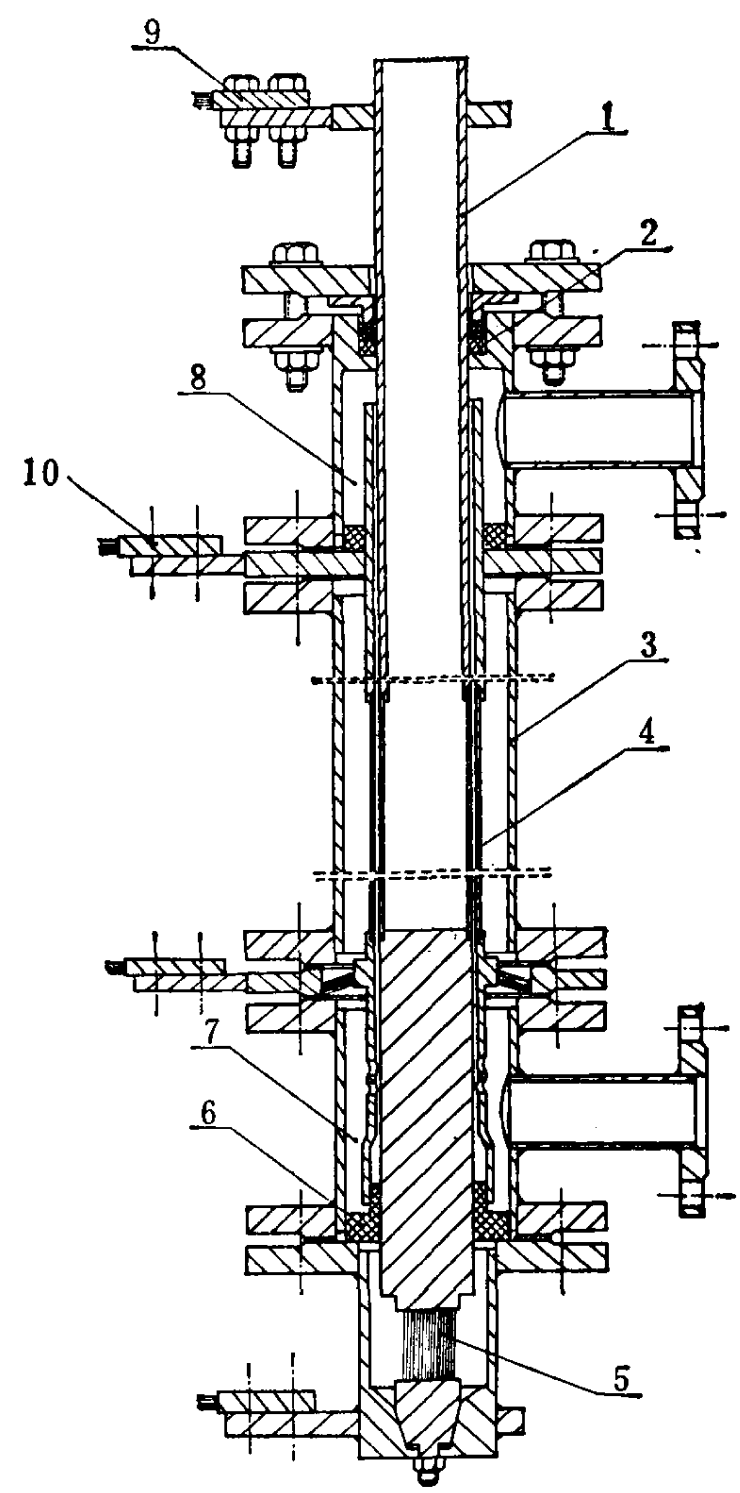

图 1 本体图

1. 内管；2、6. 定位环；3. 本体外売； 4. 外管;

5. 软铜片; 7. 出水室; 8. 进水室; $9 、 10$. 导电 铜排

量元件,靠拉杆、弹簧片,保证放置热偶结点的 聚四氟乙烯块能很好地紧贴壁面. 外套管的 热偶也全部固定在特制的聚四氟乙烯块上,

本文 1975 年 6 月 15 日收到。 
表 1 试验本体主要几何尺寸及工作参数

\begin{tabular}{|c|c|c|c|}
\hline 试验本体序号 & $1 *$ & 2 & $3^{*}$ \\
\hline 流道 曻径 $\begin{array}{ll}d_{1} & \text { (毫米) } \\
d_{2} & \text { (毫米) }\end{array}$ & $\begin{array}{l}35 \\
40\end{array}$ & $\begin{array}{l}35 \\
39\end{array}$ & $\begin{array}{l}28 \\
32\end{array}$ \\
\hline 直 径 比 $d_{2} / d_{1}$ & 1.142 & 1.112 & 1.142 \\
\hline 当量直径 $d_{e}$ (毫米) & 5 & 4 & 4 \\
\hline 元 件 材 料 & $|\mathrm{Cr}| 8 \mathrm{Ni} 9 \mathrm{Ti}$ & 黄 & $|\mathrm{Cr}| 8 \mathrm{Ni} 9 \mathrm{Ti}$ \\
\hline 元 件 壁 厚 $\delta$ (毫米) & 0.4 & 1.0 & 1.0 \\
\hline 元 件 长 度 (毫米) & 370 & 450 & 260 \\
\hline 铜稳定段长度 (毫米) & 260 & 没 & 130 \\
\hline 分电压测量距离（毫米） & 152 & 152 & 152 \\
\hline 热负荷 $q\left(\right.$ 大卡 $/$ 时 $^{\circ} \mathrm{C}$ 米 $\left.^{2}\right)$ & $4.5 \sim 12 \times 10^{3}$ & $5 \sim 7 \times 10^{5}$ & $2.8 \sim 3.3 \times 10^{6}$ \\
\hline$P_{v}$ & 2.3 & $2.3 ; 3.3$ & $1.5 \sim 3.3$ \\
\hline
\end{tabular}

安壁时使后者贴近壁面. 为了防止造成附加 电势, 热偶结点不与壁面直接接触。这样径 向热损失就可能造成外壁温的测量误差，为 此在本体外壳均匀地绕上电阻丝, 由电阻丝 通电发热, 补偿径向热损失, 保证壁温测量的 可靠、正确.

试验本体进出口水温用镍铬一考铜热电 偶测量. 热偶均预先在恒温油浴中用二级标 准水银温度计校验, 读数用 $\mathrm{P} 2 / 1$ 半自动电位 差计或 UJ9/1 型高阻电位差计, 配以 $\mathrm{AC} 9 / 2$ 型检流计测量. 电流用 0.5 级分流器 $(6000$ 安/75 毫伏配以 UJ9 型高阻电位差计测定. 电压用 UJ9 型高阻电位计配以 FJ10 型分 压 箱(采用 100:1) 以及 101 型检流计测量.

\section{二、试验结果与讨论}

我们按下式计算各截面上的放热系数

$$
\alpha_{x}=\frac{q_{F}}{\bar{t}_{w x}-t_{f x}} .
$$

其中, 热负荷按电功率计算

$$
q_{F}=\frac{0.86 I V_{\text {召 }}}{\pi D L_{\text {分 }}},
$$

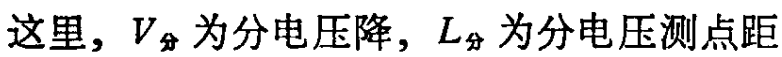
离.

由于分电压降与总电压降之间有一定的 比例关系,在预备试验里,我们确定了它的数 值. 为了方便, 试验中有时只测总电压降, 而 分电压用上述比值计算.
水温分布认为是线性的，这样对于单面 加热情况任一截面的水温为

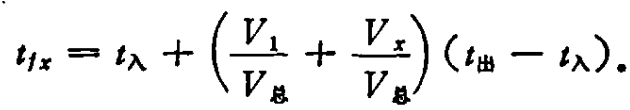

其中, $t_{\lambda}, t_{\text {出 }}$ 分别为试验段进出口水温; $V_{1} /$

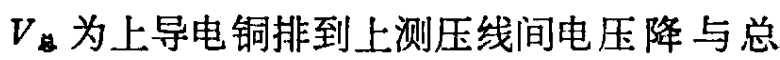

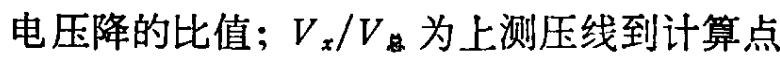
间的电压降与总电压降的比值.

而对于双面加热情况，任一截面的水温 为

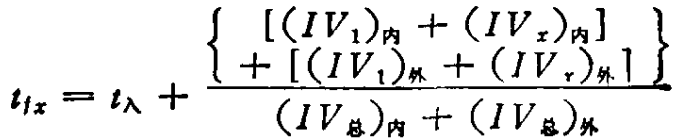

$$
\begin{aligned}
& \times\left(t_{\text {世 }}-t_{\lambda}\right) \text {. }
\end{aligned}
$$

外套管的内壁温度为

$$
\bar{t}_{w x}=\bar{t}_{o x}-\Delta t_{w x} .
$$

其中, $\bar{t}_{o x}$ 为外套管外壁的温度测量值 (周向 六个点的算术平均值), 而 $\Delta t_{w x}$ 是外套管的 壁温降,可以用热传导方程确定.

$$
\Delta t_{w x}=\frac{q_{F} D_{1}}{2 \lambda}\left[\frac{D_{2}^{2}}{D_{2}^{2}-D_{1}^{2}} \ln \frac{D_{2}}{D_{1}}-\frac{1}{2}\right] .
$$

这里 $D_{2} 、 D_{1}$ 分别为外套管的外径、内径.

内套管的外壁温度为

$$
\bar{t}_{w x}=\bar{t}_{i x}-\Delta t_{w x} .
$$

其中, $\bar{t}_{i x}$ 为内套管内壁温的平均测量值, 而

$$
\Delta t_{w x}=\frac{q_{F} D_{2}}{4 \lambda}\left[1-\frac{2 \ln D_{2} / D_{1}}{:\left(D_{2} / D_{1}\right)^{2}-1}\right] .
$$

在试验中，电功率与热功率之间的不平 衡不超过 $\pm 10 \%$,其中大多数在 $\pm 5 \%$ 之内. 
内外套管在安装时不会绝对同心，由于 偏心而造成同一截面上壁温沿周向各处不 等. 一般用无因次温度 $\theta=\left(t_{w x \max }-\bar{t}_{w x}\right) /$ $\left(\bar{t}_{w x}-t_{f x}\right)$ 来衡量偏心程度. 对于我们的试 验 $\theta \leqslant 0.12$, 所以同心度可以认为是满意的.

试验数据表明, 当 $l_{x} / d e>35$ 以后, 局 部放热系数已经达到稳定. 在整理数据时, 内外套管的数据均取自 $l / d e=67,92,60$

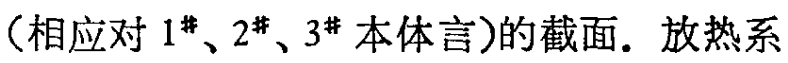
数按准则公式整理. 在图 2、图 3 表示了单 面与双面加热时的实验结果, 它们可以用下 列关系式表达.

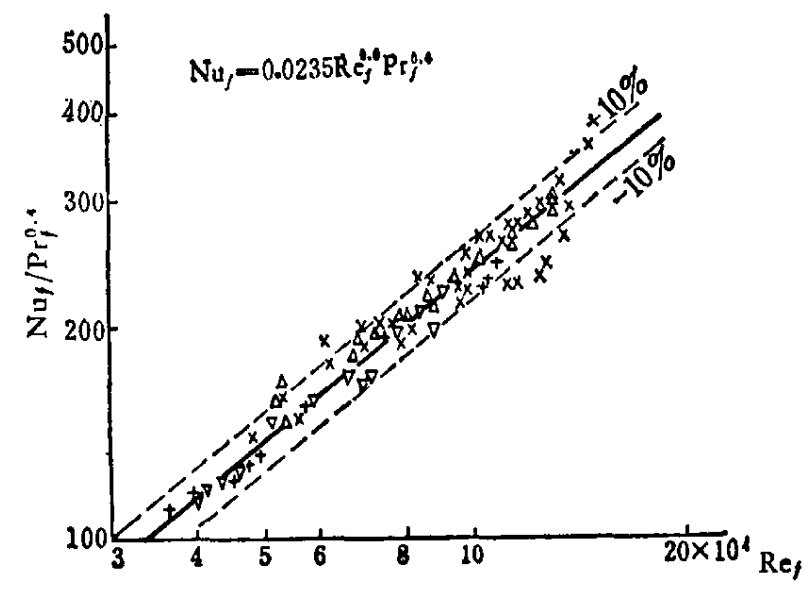

图 2 单面加热时的放热系数

$\begin{array}{cccc}\text { 符号 } & d_{2} / d_{1} & \text { 本体 } & \text { 换热面 } \\ \Delta & 1.14 & 1 * & \text { 内壁 } \\ \times & 1.14 & 1 * & \text { 外壁 } \\ \nabla & 1.11 & 2 * & \text { 内壁 } \\ + & 1.14 & 3 * & \text { 内壁 }\end{array}$

单面加热

$$
\mathrm{Nu}_{f}=0.0235 \operatorname{Re}_{f}^{0.8} \operatorname{Pr}_{f}^{0.4},
$$

双面加热

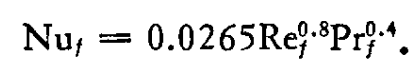

这里定性温度取的是流体平均温度. 分散度 除个别实验外不超过 $\pm 10 \%$ 。在我们试验的 热负荷范围内，热负荷对放热系数没有明显 的影响. 此外, 在图 3 上, 还列入平板双面加 热的试验数据. 从这里可以看到, 在试验范 围内 $d_{2} / d_{1}$ 的影响很小, 可以忽略.

一般情况下 $\left(q_{1} \neq q_{2} \neq 0\right)$ 的放热系数 可以根据叠加原则由极限情况 $\left(q_{1}=0, q_{2}=\right.$ 0 ) 计算 ${ }^{[2]}$.

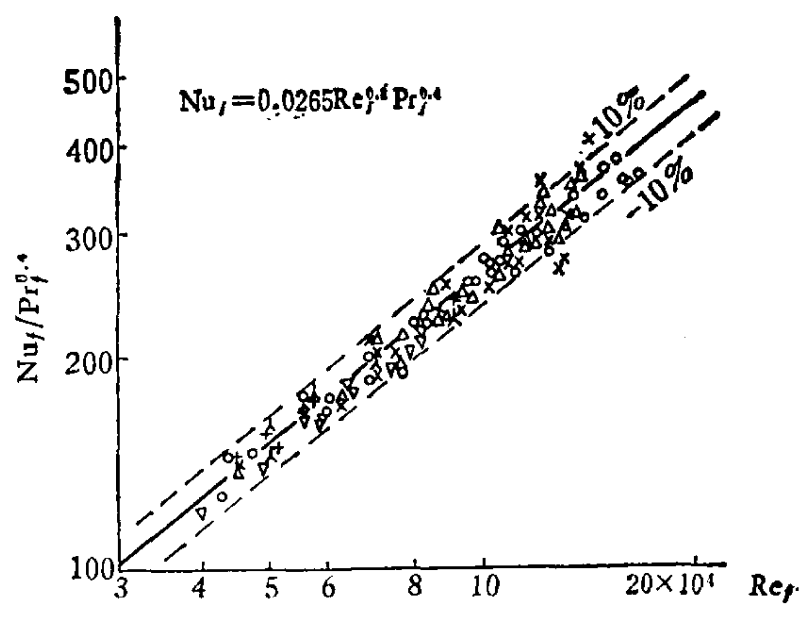

图 3 双面加热时的放热系数

$\begin{array}{cccc}\text { 符号 } & d_{2} / d_{1} & \text { 本体 } & \text { 换热面 } \\ \Delta & 1.14 & 1 * & \text { 内壁 } \\ \times & 1.14 & 1 * & \text { 外壁 } \\ \nabla & 1.11 & 2 * & \text { 内壁 } \\ + & 1.14 & 3 & \text { 内壁 } \\ \hat{\lambda} & 1.14 & 3 * & \text { 外壁 } \\ 0 & 1.00 & \text { 平板 } & \end{array}$

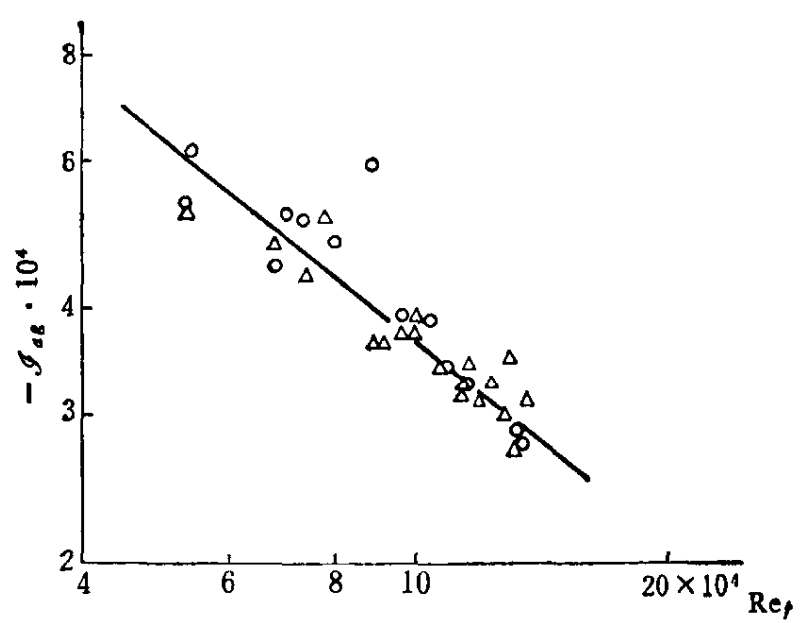

图 4 无因次绝热温度 $\mathscr{I}_{a g}$ 与 $\mathrm{Re}$, 的关系

$$
\begin{gathered}
(\operatorname{Pr} \approx 2.4) \\
\Delta \mathscr{I}_{\text {Iag }} ; \quad \circ \mathscr{I}_{2 a \mathrm{~g}}
\end{gathered}
$$

$$
\begin{aligned}
\mathrm{Nu}_{1} & =\frac{\mathrm{Nu}_{1 H}}{1+q_{2} / q_{1} \mathscr{I}_{1 a g} \mathrm{Nu}_{1 H}}, \\
\mathrm{Nu}_{2} & =\frac{\mathrm{Nu}_{2 H}}{1+q_{1} / q_{2} \mathscr{I}_{2 a g} \mathrm{Nu}_{2 H}}
\end{aligned}
$$

这里,

$$
\begin{gathered}
\mathrm{Nu}_{1 H}=\frac{\alpha_{1 H} d_{e}}{\lambda}=\frac{q_{1}\left(d_{2}-d_{1}\right)}{t_{w 1}^{\prime \prime}-\bar{t}^{\prime \prime}}, \\
\mathrm{Nu}_{2 H}=\frac{\alpha_{2 H} d_{e}}{\lambda}=\frac{q_{2}\left(d_{2}-d_{1}\right)}{t_{w 2}^{\prime}-\bar{t}^{\prime}}, \\
\mathscr{I}_{1 a g}=\frac{\left(t_{w_{1}}^{a g}-\bar{t}^{\prime}\right) \lambda}{q_{2} d_{c}},
\end{gathered}
$$




$$
\mathscr{I}_{2 a g}=\frac{\left(t_{w_{2}}^{a b}-\bar{t}^{\prime}\right) \lambda}{q_{1} d_{e}} \text {. }
$$

在 1 本体试验中, 我们还测量了无因次 绝热温度 $\mathscr{I}_{\text {ag }}$. 由图 4 可见 $\mathscr{I}_{1 a g}, \mathscr{I}_{2 a g}$ 与 $\mathrm{Re}^{0.8}$ 成反比,它们可表成

$$
\mathscr{I}_{1 a g}=\mathscr{I}_{2 a g}=-3.6 \mathrm{Re}^{-0.8} \text {. }
$$

根据 Петухов 的测量 ${ }^{[3]}$ (用的工质是空 气), 比较式(11)与他推荐的经验公式, 可以 看出 $\mathscr{I}$ 还与 $\operatorname{Pr}$ 数有关. 我们用 $\operatorname{Pr}^{n}$ 来表示 这个关系,这样就可以得到

$$
\mathscr{I}_{a g} \mathrm{Nu}_{H}=-0.183 \operatorname{Pr}^{-0.43} \text {. }
$$

所以一般情况下的放热系数可写作

$$
\mathrm{Nu}_{1}=\frac{\mathrm{Nu}_{H}}{1-0.183 \mathrm{Pr}^{-0.43} q_{2} / q_{1}}
$$

$$
\text { 或 } \mathrm{Nu}_{1}=\frac{0.0235 \mathrm{Re}^{0.8} \mathrm{Pr}^{0.4}}{1-0.183 \mathrm{Pr}^{-0.43} q_{2} / q_{1}} \text {. }
$$

对于双面加热 $q_{2} / q_{1}=1$, 由上式得(取 $\operatorname{Pr}=$ 2.4)

$$
\mathrm{Nu}_{\text {双 }}=0.0269 \mathrm{Re}^{0.8} \mathrm{Pr}^{0.4},
$$

与式(8)相差不超过 $2 \%$. 此外式(12)与我们 的理论解 ${ }^{[1]}$ 也是非常接近的.

\section{参考资 料}

[1] 颜迪民,机械工程学报, 12 1964, 3, 17-41.

[2] Петухов Б. С. и Ройзен. Л. И., Инжемернофизический журнал., 1963, 3, 3-11.

[ 3 ] Петухов Б. С. и Ройзен Л. И., Теплофизика Высоких Температ, 1 (1963), 3, 416-424.

\section{$d l$-喜 树 碱 的 全 合 成 \\ 上海第五制药厂上海第十二制药厂 上海医药工业研究院上海药物研究所}

喜树 (Camptotheca acuminata) 是珙桐科 (Nyssaceae) 乔木, 为我国所特有. 华南各省 分布较广, 资源丰富, 1966 年 Wall 等从喜 树中分离得抗肿瘤有效成分喜树碱 (Camptothecin, I), 并确定其化学结构 ${ }^{[1]}$. 喜 树碱是 一种含有五个环的化合物, 结构较为特殊, 具 有明显的抗肿瘤活性. 因此引起许多合成工 作者的注意, 并先后发表了不少关于全合成 成功的报道 ${ }^{[2]}$. 上海药物研究所于 1969 年亦 分离得喜树碱 ${ }^{[3]}$, 临床试用结果表明, 喜树碱 对胃癌、直肠结肠癌、膀胱癌等恶性肿瘤具有 一定的治打作用，但其毒性较大。鉴于喜树

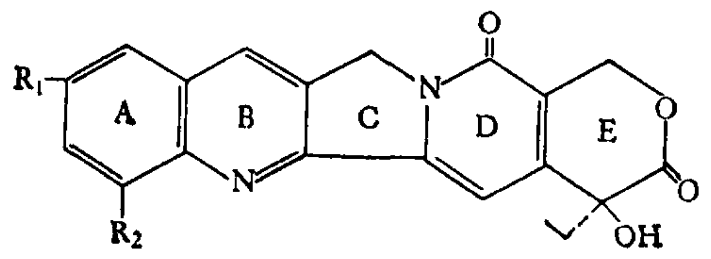

I. $\mathrm{R}_{1}=\mathrm{H}, \mathrm{R}_{2}=\mathrm{H}$; II. $\mathrm{R}_{1}=\mathrm{OH}, \mathrm{R}_{2}=\mathrm{H}$; III. $\mathbf{R}_{\mathbf{1}}=\mathrm{H}, \mathrm{R}_{\mathbf{2}}=\mathrm{Cl}$
碱的一些 $\mathrm{A}$ 环类似物 (如 II, III) 在动物试验 中具有较喜树碱毒性小、抗㾇谱广的特点.因 此, 寻找一种具有生产价值, 适用于喜树碱及 其类似物的全合成方法，有着很大的意义.

本文报道的下列全合成方法,步骤较短,

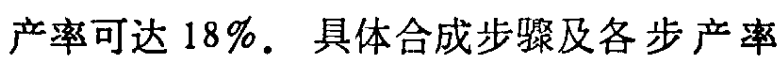
如式 IV-XIII 所示.

由 3-氯基-4-甲基-6-乙氧炭基-2(1H)吡啶酮（IV）在二甲基甲酰胺溶液中与丙 烯酸甲酯经缩合、环合得 $\mathrm{V}\left[250^{\circ} \mathrm{C}\right.$ 分解 ( $80 \%$ 乙酸), 产率 $80 \%$ ]. V 经盐酸水解脱 羒后得 VI $\left[219^{\circ} \mathrm{C}\right.$ 分解 (丙酮), 产率 $\left.90 \%\right]$. VI 用乙二醇保护酩基得缩酮 VII[ 191一 $192{ }^{\circ} \mathrm{C}$ 分解 (碳酸二乙酯)，产率 $93 \%$ I.VII 在甲苯溶液中, 氢化钠存在下与碳酸二乙酯 缩合得 VIII $172^{\circ}-173^{\circ} \mathrm{C}$ (甲醇), $76 \%$ ].VIII 经碘乙烷乙基化得 $\operatorname{IX}\left[122^{\circ}-123^{\circ} \mathrm{C}\right.$ (乙醇),

本文 1975 年 8 月 11 日收到。 